\title{
The Clustering Coefficient of Multiple Parallel Airlines AANET
}

\author{
Xue Liu ${ }^{[1],[2]}$, Xiaoping Zeng ${ }^{[1]}$, Zhiming Wang ${ }^{[1]}$, Bin Zhu ${ }^{[1]}$ and Li Chen ${ }^{[1]}$ \\ [1]College of Communication Engineering, Chongqing University, 400030, \\ China. \\ [2] Chongqing Communication Institute, Chongqing, 400035, China.
}

\begin{abstract}
As a kind of ad hoc networks, aeronautical ad hoc networks (AANET) has its own characteristic, which is that nodes in the networks often move along multiple specified lines. Considering this characteristic, we deal with parallel airlines in different height levels of the airspace and work out the clustering coefficient of the AANET, which is one of the key metrics of a complex networks. For two parallel airlines scenario, we give an accurate calculation of its clustering coefficient. For multiple airlines scenario, though accurate calculation can be done by extending the method for two parallel airlines, it needs too much computation. Thus, we give an approximate calculation for the scenario, which is much simpler and the deviation becomes very small when the number of airlines becomes large as shown in numeric results.
\end{abstract}

Keywords: AANET, complex networks, clustering coefficient, parallel airlines

\section{Introduction}

With the rapid development of civil aviation, the demands of accessing Internet on board have become stronger than ever before. However, in polar, desert, marine and other areas where base stations can't be built, aircrafts have to use satellite links or large span multi-hop links within AANET to reach base stations. Due to the large delay, high cost and limited bandwidth of satellite links, AANET is preferred for Internet accessing in these regions [1-2].

For AANET, the Newsky project has proposed networking strategies with mobile IPv6 technology [3]. ATENAA project has studied Ka-band array antennas and optical communications between aircrafts [4]. Justin R, Abdul $\mathrm{J}$ et al have presented cross-layer networking solutions among physical, mac, network and transport layers [5]. Quentin Vey et al have proposed the use of Direct Sequence CDMA (DS-CDMA) at the access layer [2] while Jianshu Yan et al have studied the capacity of single flight path AANET [6] .

As a kind of ad hoc networks, AANET has its own characteristic. Nodes in AANET often move along multiple specified lines, which behave not like the nodes in other ad hoc networks where they can change moving directions at any time. There have been a lot of works focused on the normal ad hoc networks and some of them have been done by using the theory of complex networks [7-10]. Especially, for clustering coefficient, which is one of the key metrics of complex networks [11], refs [12-15] have given some deep researches. However, all of them are focus on networks where nodes distribute in a plan while nodes in AANET are distributed along some specified lines, which is one of its most important characteristics. Therefore, in this paper, we focus on some kind of AANET and work out its clustering coefficient.

\section{Assumptions}

According to the specifications of the International Civil Aviation Organization ICAO, the airspace is divided into several height levels and the distance between two adjacent 
levels is at least $300 \mathrm{~m}$. In this paper, we deal with one single air route with several different height levels(call them parallel airlines), which has the same scenario as in reference [6], and work out the clustering coefficients of two parallel airlines AANET and multiple parallel airlines AANET respectively by ignoring the border effect.

Since AANET is not deployed currently and none of the achievements mentioned in paragraph two of this section is taken as standard or in real use, we don't consider the physical and access layers of AANET and use a fixed value, $r$, as the communication range for all planes. As many other works do, we assume the spatial distribution of planes on each airline follows Poisson point process and, for simplicity, we assign a same plane density to all the airlines.

\section{Clustering Coefficient of Two Parallel Airlines AANET}

If we consider a node and its neighbors in networks, the probability that two of these neighbors are connected is defined as clustering coefficient of the network[16]. In our scenario, we denote $p(b \leftrightarrow c \mid a \leftrightarrow b, a \leftrightarrow c)$ as the clustering coefficient of the parallel airlines AANET, which is the probability that the distance between planes ' $b$ ' and ' $c$ ' is no more than the plane's communication range, $r$, under the condition that they are both the neighbors of plane ' $\mathrm{a}$ '. In the following context, we use $P_{c c}$ to represent $p(b \leftrightarrow c \mid a \leftrightarrow b, a \leftrightarrow c)$ for short.

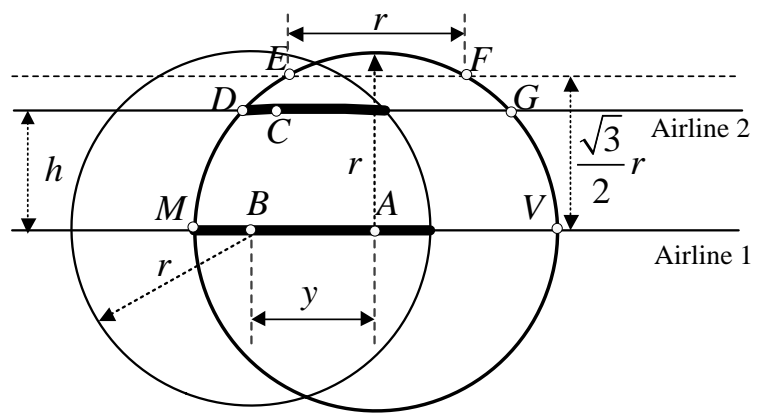

Figure 1. Two Parallel Airlines

In this part, we consider the AANET of only two parallel airlines. In Fig.1, two parallel airlines named Airline 1 and Airline 2 respectively have a distance $h$ between them. We set plane 'a' at point $A$ of Airline 1, the circular communication coverage of which covers segment $M V$ of Airline 1 and segment $D G$ of Airline 2. Plane 'b' and plane 'c' are somewhere in $M V$ and $D G$. In the following context, if we say "a plane covers something (point or segment)", it means "something is under the circular communication coverage of a plane".

When plane ' $\mathrm{b}$ ' is at point $B$, the overlapping segments of airlines covered both by plane ' $a$ ' and plane ' $b$ ' can be represented by the bold lines in Fig.1. Since planes are uniformly distributed in airlines, which is one of the properties of Poisson point process, the probability that the distance between planes ' $b$ ' and ' $c$ ' is no more than $r$ is the ratio of the total length of the overlapping segments (denote it as $l(y)$, where $y$ can be looked as the location of plane ' $b$ ' for now) to the total length of covered segments by plane ' $a$ ' (denote it as $L$ ). Then, by denoting $A(y) d y$ as the probability that plane ' $\mathrm{b}$ ' is at location $y$, we get the expression of the clustering coefficient in Eq.1.

$$
P_{c c}=\int A(y)\left[\frac{l(y)}{L} d y\right.
$$

Since plane 'b' can be at any point in $D G$ or $M V$ and the distance $h$ can vary from 0 to $r$, there is no unified form for $A(y)$ aid $l(y)$. Thus, we let $A_{s e g}(y)$ and $l_{\text {Seg }}(y)$, where 
'Seg' stands for one of the segments covered by plane 'a', represent $A(y)$ and $l(y)$ respectively when plane ' $b$ ' is on that 'Seg'. Then, Eq.1 can be written as Eq.2.

$$
P_{c c}=\int_{M V} A_{M V}(y)\left[\frac{l_{M V}(y)}{L} d y+\int_{D G} A_{D G}(y)\left\lfloor\frac{l_{D G}(y)}{L} d y\right.\right.
$$

According to where plane ' $\mathrm{b}$ ' is and the value range of $h$, we classify the expressions of $A(y)$ and $l(y)$ in Table.1, in which the location of plane ' $b$ ', $y$, is the distance between plane ' $a$ ' and plane ' $b$ '. The derivation for each expression is in the following context.

Table 1. Classifications For $A(y)$ and $l(y)$

\begin{tabular}{|c|c|c|c|c|}
\hline & $\begin{array}{l}\text { Segment } \\
\text { where } \\
\text { plane ' } b \text { ' is }\end{array}$ & $\begin{array}{l}\text { Value } \\
\text { Range } \\
\text { Of } h\end{array}$ & $A(y)$ & $l(y)$ \\
\hline 1 & $M V$ & $0<h \leq \frac{\sqrt{3}}{2} r$ & $\frac{1}{L}, \quad 0<y<r$ & $L-2 y, \quad 0<y<r$ \\
\hline 2 & $M V$ & $\frac{\sqrt{3}}{2} r<h<r$ & $\frac{1}{L}, \quad 0<y<r$ & $\begin{array}{l}L-2 y, \quad 0<\mathrm{y}<2 \sqrt{r^{2}-h^{2}} \\
2 r-y, \quad 2 \sqrt{r^{2}-h^{2}}<\mathrm{y}<\mathrm{r}\end{array}$ \\
\hline 3 & $D G$ & $0<h \leq \frac{\sqrt{3}}{2} r$ & $\frac{y}{L \sqrt{y^{2}-h^{2}}}, \quad h<y<r$ & $\begin{array}{l}4 \sqrt{r^{2}-h^{2}}, h<y<\sqrt{2 r^{2}-2 r \sqrt{r^{2}-h^{2}}} \\
L-2 \sqrt{y^{2}-h^{2}}, \sqrt{2 r^{2}-2 r \sqrt{r^{2}-h^{2}}}<y<r\end{array}$ \\
\hline 4 & $D G$ & $\frac{\sqrt{3}}{2} r<h<r$ & $\frac{y}{L \sqrt{y^{2}-h^{2}}}, \quad h<y<r$ & $4 \sqrt{r^{2}-h^{2}}, \quad h<y<r$ \\
\hline
\end{tabular}

\subsection{Classification 1}

Since plane ' $\mathrm{b}$ ' is in the same airline with plane ' $\mathrm{a}$ ', when y changes $d y$, plane ' $\mathrm{b}$ ' moves $d y$ too. In the addition of the condition that planes are uniformly distributed in airlines, we obtain

$$
\begin{aligned}
& A(y)=\frac{1}{L}, \quad 0<y<r \\
& L=2 r+2 \sqrt{r^{2}-h^{2}}
\end{aligned}
$$

When $h$ is no more than $(\sqrt{3} / 2) r$, the length of segment $D G$ is always no less than $r$. That means there is always an overlapping segment in $D G$ and the length of it changes linearly with $y$. Thus, it is easy to get

\subsection{Classification 2}

$$
l(y)=L-2 y, \quad 0<y<r
$$

$A(y)$ is the same as in Classification 1 . When $h$ is between $(\sqrt{3} / 2) r$ and $r$, the length of $D G$ is always less than $r$. That means, if $y$ is greater than a certain value, there may be no overlapping segment in $D G$. It's easy to find that certain value is $2 \sqrt{r^{2}-h^{2}}$. Thus, we can get

$$
l(y)= \begin{cases}L-2 y, & 0<\mathrm{y}<2 \sqrt{r^{2}-h^{2}} \\ 2 r-y, & 2 \sqrt{r^{2}-h^{2}}<\mathrm{y}<\mathrm{r}\end{cases}
$$




\subsection{Classification 3}

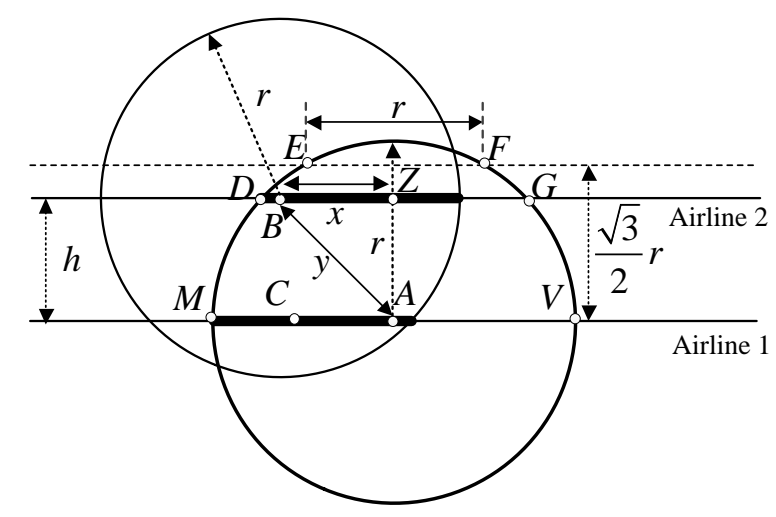

Figure 2. Plane 'a' And Plane 'b' being On Different Airlines

In Fig.2, point $Z$ represents the vertical projection point of $A$ on segment $D G$ and $x$ denotes the distance between plane ' $b$ ' and point $Z$. Since plane ' $b$ ' is not in the same airline with plane ' $\mathrm{a}$ ', when $y$ changes $d y$, plane ' $\mathrm{b}$ ' no longer moves $d y$ as it does in Classification 1 and Classification 2. Thus, we have the following derivation,

$$
\begin{aligned}
& \because x^{2}=y^{2}-h^{2} \\
& \therefore 2 x=2 y \frac{d y}{d x} \\
& \therefore d x=\frac{y}{\sqrt{y^{2}-h^{2}}} d y \\
& \therefore A(y)=\frac{y}{L \sqrt{y^{2}-h^{2}}}, \quad h<y<r
\end{aligned}
$$

As seen in Fig.2, the value range of $y$ is now from $h$ to $r$ and, when $y$ is greater than a certain value, the whole segment $D G$ is a part of the overlapping segments. That certain value is $\sqrt{2 r^{2}-2 r \sqrt{r^{2}-h^{2}}}$ and we get

$$
l(y)=\left\{\begin{array}{cc}
4 \sqrt{r^{2}-h^{2}}, & h<y<\sqrt{2 r^{2}-2 r \sqrt{r^{2}-h^{2}}} \\
L-2 \sqrt{y^{2}-h^{2}}, & \sqrt{2 r^{2}-2 r \sqrt{r^{2}-h^{2}}}<y<r
\end{array}\right.
$$

\subsection{Classification 4}

$A(y)$ is the same as it in Classification 3. When $h$ is between $(\sqrt{3} / 2) r$ and $r$, the length of $D G$ is always less than $r$ and the whole segment is a part of the overlapping segments. Thus, we obtain

$$
l(y)=4 \sqrt{r^{2}-h^{2}}, \quad h<y<r
$$

Therefore, according to Table. 1 and Eq. 2 and considering that each segment covered by plane 'a' is bilaterally symmetrical (e.g., $M V$ is bilaterally symmetrical about point $A$ ), we get two expressions of the clustering coefficient due to the two different value ranges of $h$.

For $0<h \leq \frac{\sqrt{3}}{2} r$, 


$$
\begin{aligned}
P_{c c}= & 2 \int_{0}^{r} \frac{1}{L} \frac{L-2 y}{L} d y+2 \int_{h}^{\sqrt{2 r^{2}-2 r \sqrt{r^{2}-h^{2}}}} \frac{y}{L \sqrt{y^{2}-h^{2}}} \frac{4 \sqrt{r^{2}-h^{2}}}{L} d y \\
& +2 \int_{\sqrt{2 r^{2}-2 r \sqrt{r^{2}-h^{2}}}}^{r} \frac{y}{L \sqrt{y^{2}-h^{2}}} \frac{L-2 \sqrt{y^{2}-h^{2}}}{L} d y \\
= & \frac{4 \sqrt{r^{2}-h^{2}}}{L^{2}}(4 r-1)
\end{aligned}
$$

For $\frac{\sqrt{3}}{2} r<h<r$,

$$
\begin{aligned}
P_{c c}= & 2 \int_{0}^{2 \sqrt{r^{2}-h^{2}}} \frac{1}{L} \llbracket \frac{L-2 y}{L} d y+2 \int_{2 \sqrt{r^{2}-h^{2}}}^{r} \frac{1}{L}\left[\frac{2 r-y}{L} d y\right. \\
& +2 \int_{h}^{r} \frac{y}{L \sqrt{y^{2}-h^{2}}} \frac{4 \sqrt{r^{2}-h^{2}}}{L} d y \\
= & \frac{6}{L^{2}}\left(2.5 r^{2}-2 h^{2}\right)
\end{aligned}
$$

\section{Clustering Coefficient of Multiple Parallel Airlines AANET}

As seen in Fig.3, there are several parallel airlines with uniformly interval, $h$, between adjacent two. Plane ' $a$ ' and plane ' $b$ ' both can cover several airlines and the number of the overlapping segments covered by them is due to the values of $h$ and $r$. Since there is no restriction on $r$, the number of overlapping segments can be large and that means the number of the terms on the right side of Eq.2 can be large too. For each term, we must split the integral interval according to the value range of $h$ just as what we have done in section II. However, this time, the number of the value ranges of $h$ is not only two and it varies in accordance with $r$. Thus, it needs too much computation to give an accurate calculation for the clustering coefficient in multiple parallel airlines AANET, though it can be done. Thus, we give a simpler, approximate calculation by the following way.

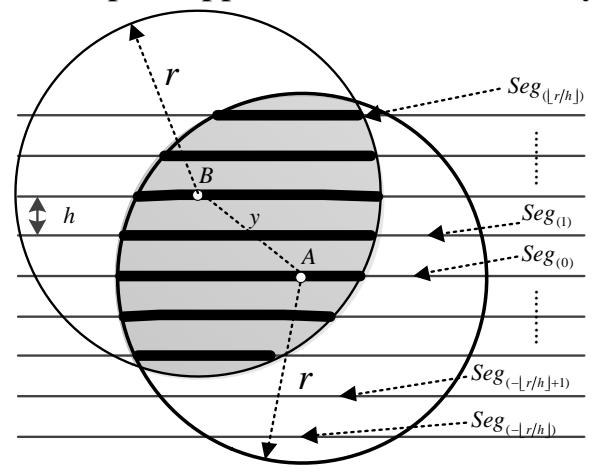

Figure 3. Multiple Parallel Airlines

Let us first consider the area of the shadow region in Fig.3. We use $y$ to denote the distance between plane ' $\mathrm{a}$ ' and ' $\mathrm{b}$ ' again and $S(y)$ to denote the area of the shadow region, which is given below

$$
S(y)=r^{2}\left(2 \arccos \left(\frac{y}{2 r}\right)-\sin \left(2 \arccos \left(\frac{y}{2 r}\right)\right)\right)
$$

We can redraw some items in Fig. 3 to Fig. 4 and use the area of the shadow region in Fig. 4 to approximate the area of the shadow region in Fig.3. Then, by assuming there are $n(n \geq 2)$ overlapping segments, denoting $l_{k}(y)(k=1,2,3 \ldots . . n)$ as the length of each segment and defining $l(y)$ as the total length of all the overlapping segments, we obtain 


$$
S(y) \approx \sum_{k=1}^{n} h l_{k}(y)=h l(y)
$$

In a similar way, if $L_{\text {all }}$ denotes the total length of the covered segments by plane 'a', we can get

$$
\begin{aligned}
& \pi r^{2} \approx h L_{\text {all }} \\
& L_{\text {all }}=2 r+\sum_{i}^{\lfloor r / h\rfloor} 4 \sqrt{r^{2}-(i h)^{2}}
\end{aligned}
$$

Therefore, we obtain

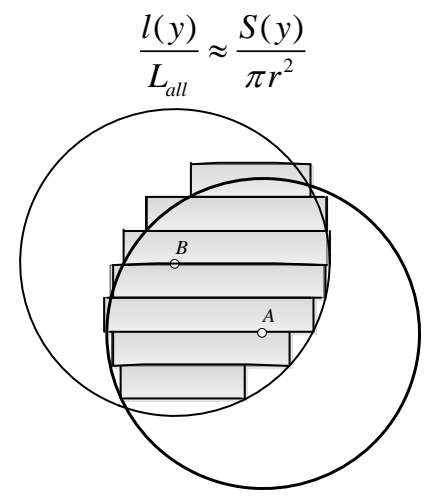

Figure 4. Simplified Figure

In fact, there are $\lfloor r / h\rfloor+1$ segments covered by plane 'a'. As shown in Fig.3, we mark these segments with $\mathrm{Seg}_{(g)}$, where ' $g$ ' is an integer and it varies from $-\lfloor r / h\rfloor$ to $\lfloor r / h\rfloor$. Then, according to Eq.2, we can get the approximate clustering coefficient of multiple parallel airlines AANET in Eq.5.

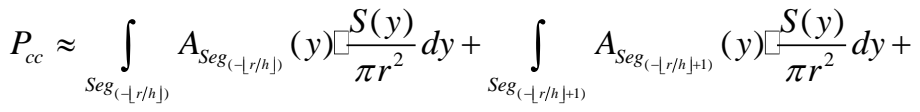

$$
\begin{aligned}
& \ldots \ldots+\int_{S e g_{([r / h])}} A_{S e g_{(\lfloor r / h)}}(y)\left[\frac{S(y)}{\pi r^{2}} d y\right.
\end{aligned}
$$

Since $S e g_{(-g)}$ and $S e g_{(g)}$ are symmetrical about $S e g_{(0)}$, Eq.6 can be written as Eq.7.

$$
P_{c c} \approx \int_{\operatorname{Seg}_{(0)}} A_{S e g_{(0)}}(y) \leftarrow \frac{S(y)}{\pi r^{2}} d y+\sum_{i=1}^{\lfloor r / h\rfloor} \int_{\operatorname{Seg}_{(i)}} 2 A_{S e g_{(i)}}(y) \frac{S(y)}{\pi r^{2}} d y
$$

Then, when plane ' $b$ ' is in segment $\operatorname{Seg}_{(0)}$, as what we do in the Classification 1 in section II, we can get

$$
A_{\text {Seg }_{(0)}}(y)=\frac{1}{L_{\text {all }}}, \quad 0<y<r
$$

Since all the segments covered by plane 'a' are bilaterally symmetrical, we obtain

$$
\int_{S e g_{(0)}} A_{S e g_{(0)}}(y) \llbracket \frac{S(y)}{\pi r^{2}} d y=2 \int_{0}^{r} \frac{1}{L_{\text {all }}} \backsim \frac{S(y)}{\pi r^{2}} d y
$$

When plane ' $\mathrm{b}$ ' is in segment $\operatorname{Seg}_{(i)}(i=1,2,3 \ldots\lfloor r / h\rfloor)$, we can easily get $A_{\operatorname{Seg}_{(i)}}(y)$ according to the derivation in Classification 3 in section II.

Then, we obtain

$$
A_{\operatorname{Seg}_{(i)}}(y)=\frac{2 y}{L_{\text {all }} \sqrt{y^{2}-(i h)^{2}}}, \quad i h<y<r
$$




$$
\sum_{i=1}^{\lfloor r / h\rfloor} \int_{\operatorname{Seg}_{(i)}} 2 A_{\operatorname{Seg}_{(i)}}(y)\left[\frac{S(y)}{\pi r^{2}} d y=2 \sum_{i=1}^{\lfloor r / h\rfloor} \int_{i h}^{r} \frac{2 y}{L_{\text {all }} \sqrt{y^{2}-(i h)^{2}}} \frac{S(y)}{\pi r^{2}} d y\right.
$$

Therefore, we get the clustering coefficient of the networks in Eq.7.

$$
\begin{aligned}
P_{c c} & \approx 2 \int_{0}^{r} \frac{1}{L_{\text {all }}}\left[\frac{S(y)}{\pi r^{2}} d y+2 \sum_{i=1}^{\lfloor r / h\rfloor} \int_{i h}^{r} \frac{2 y}{L_{\text {all }} \sqrt{y^{2}-(i h)^{2}}} \frac{S(y)}{\pi r^{2}} d y\right. \\
& \approx \frac{r}{3 \pi L_{\text {all }}}(16-6 \sqrt{3})+\frac{2}{3}-\frac{\sqrt{3}}{2 \pi}+\frac{8}{\pi r^{2} L_{\text {all }}} \sum_{i=1}^{\left\lfloor\frac{r}{h}\right\rfloor} \int_{i h}^{r} \sqrt{y^{2}-(i h)^{2}} \square \sqrt{r^{2}-(\mathrm{y} / 2)^{2}} d y
\end{aligned}
$$

\section{Numeric Results}

In simulation, we generate planes on each parallel airline according to Poisson point process. After delete the planes at the borders, which can't cover $2\lfloor r / h\rfloor+1$ segments, we calculate every remaining plane's clustering coefficient by the following way. First, for each plane, we count the number of its neighbors and denote it as $k$. Second, for every pair of its neighbors, we check if their distance is less than the communication range $r$. If yes, we add ' 1 ' to another integer, $K$, whose initial value is zero. After checking all the pairs, we get the plane's clustering coefficient, which is $2 K /(k \llbracket(k-1)), k \geq 2$. When all the remaining planes' clustering coefficients are worked out, we take an average of them and the result is the clustering coefficient of the networks ignoring the border effect.

Table 2. Parameters for Simulations

\begin{tabular}{ccc}
\hline & Two Parallel Airlines & Multiple Parallel Airlines \\
& Scenario & Scenario \\
\hline Airline Length & $1000 \mathrm{~km}$ & $1000 \mathrm{~km}$ \\
Plane Density & $1 / \mathrm{km}, 5 / \mathrm{km}$ or & $3 / \mathrm{km}, 6 / \mathrm{km}$ or \\
Airlines' Number & $10 / \mathrm{km}$ & $9 / \mathrm{km}$ \\
$r$ & 2 & 100 \\
$h$ & $3 \mathrm{~km}$ & $5 \mathrm{~km}$ \\
& $0.05 r, 0.1 r, 0.15 r \ldots$, & $0.05 r, 0.1 r, 0.15 r \ldots, \quad$ or \\
\hline
\end{tabular}

Some parameters in simulations are listed in Table.2. Fig.5 shows the theoretical and simulation results for two parallel airlines scenario while Fig.6 is for multiple parallel airlines scenario. Since the calculation for two parallel airlines scenario is accurate and has nothing to do with the plane densities in airlines, the theoretical and simulation values in Fig. 5 are almost the same and we only draw the simulation value with plane density 5 planes $/ \mathrm{km}$. Similarly, the deviation in Fig.6 is due to approximate calculation and, when $h$ becomes smaller, which means a plane can cover more segments, the deviation becomes smaller too.

To verify that the plane density has nothing to do with the clustering coefficients in our scenarios, we do the same simulations with different plane densities again and the results for two parallel airlines and multiple parallel airlines scenarios are shown in Fig.7 and Fig. 8 respectively. Note that the values in Fig. 6 and Fig. 8 are very close to that in ref [12], which has studied the networks with nodes distributed uniformly in a plan. Especially when $h / r$ is small, which means airlines are close to each other, the planes in airlines can be looked like uniformly distributed in a plan. 


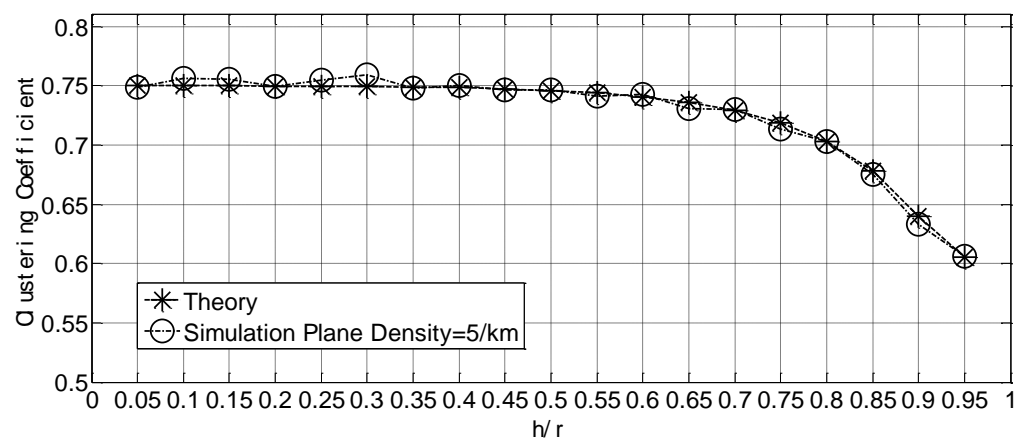

Figure 5. Results for Two Parallel Airlines Scenario

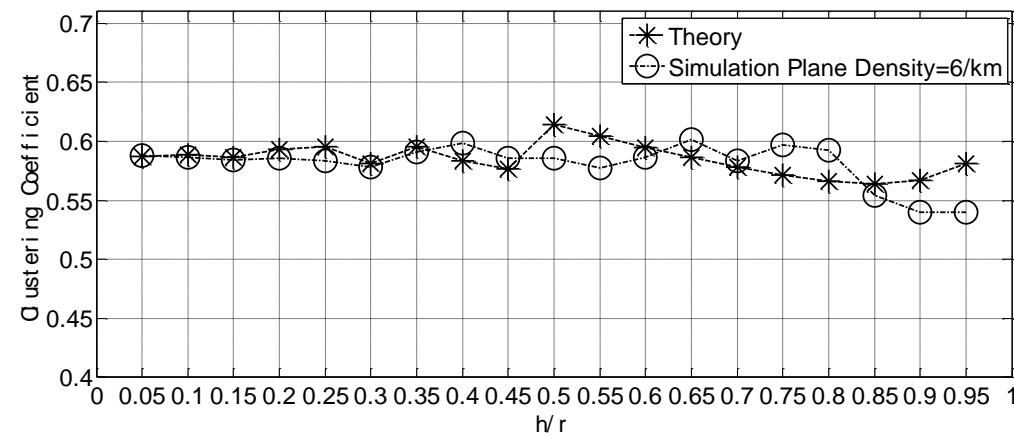

Figure 6. Results for Multiple Parallel Airlines Scenario

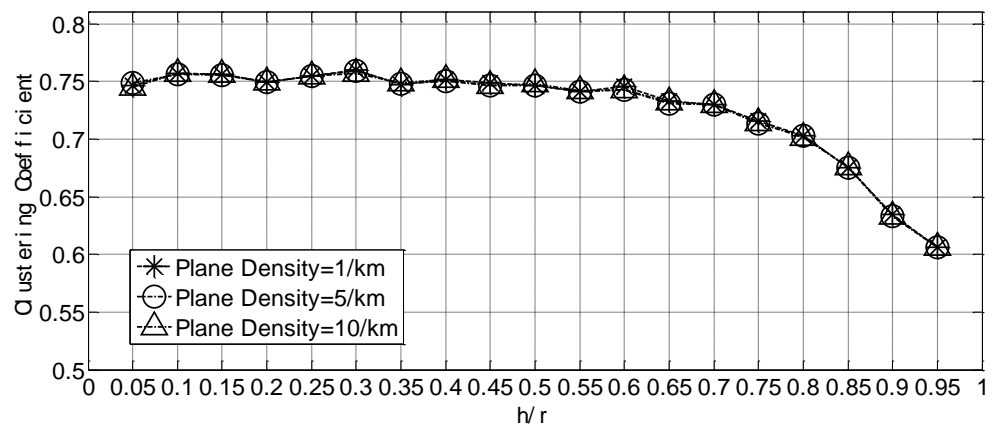

Figure 7. Two Parallel Airlines Scenarios With Different Plane Densities

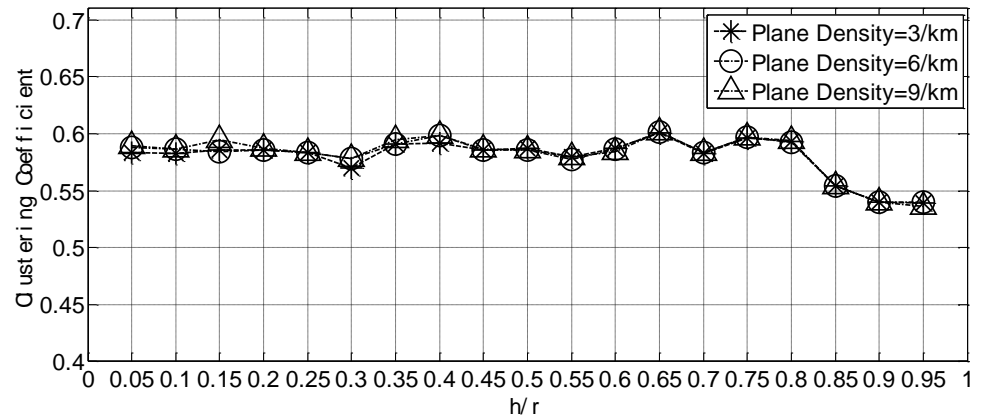

Figure 8. Multiple Parallel Airlines Scenarios With Different Plane Densities 


\section{Conclusion}

As a kind of ad hoc networks, AANET has its own characteristic, which is that nodes in the networks often move along multiple specified lines. Considering this characteristic, we deal with parallel airlines in different height levels of the airspace and work out the clustering coefficient of the AANET of parallel airlines. From the simulation, which verifies the correctness of our work, we can find that the plane densities of the airlines are not important as the ratio of the interval of two airlines and the communication range of plane, if the densities are the same. In fact, if we assign different plane densities to different airlines, the derivation of the clustering coefficient is almost the same, but the expression of it will be more complicated.

\section{Acknowledgment}

Our research is supported by the National Natural Science Foundation of China (Grant No. 61171089, 91438104 and 61302054), Chongqing Science \& Technology Commission (Grant No. cstc2014yykfA40002) and the 2013 Innovative Team Construction Project of Chongqing Universities.

\section{References}

[1] E. Sakhaee and A. Jamalipour, IEEE Journal on Selected Areas in Communications, vol. 24, no. 9, (2006).

[2] Q. Vey, A. Pirovano, J. Radzik and F. Garcia, "Aeronautical Ad Hoc Network for Civil Aviation", Communication Technologies for Vehicles Lecture Notes in Computer Science, (2014).

[3] M. Schnell and S. Scalise, "IEEE Aerospace and Electronic Systems Magazine", vol. 22, no. 5, (2007).

[4] K. Karras, "Aeronautical Mobile Ad Hoc Networks. The 14th European Wireless Conference", (2008).

[5] R. Justin and J. Abdul, "IEEE Transactions on Aerospace and Electronic Systems", vol. 47, no. 4, (2011).

[6] J. Yan, C. Hua, C. Chen and X. Guan, Wireless Networks, vol. 21, no. 3, (2014).

[7] N. Sarshar and V. Roychowdhury, Physical Review E. 69.026101, (2004).

[8] Y. Hayashi and J. Matsukubo, Physical Review E.73.066113, (2006).

[9] L. Wang, C. P. Zhu and Z. M. Gu, Physical Review E.78.066107, (2008).

[10] E. Estrada, Physical Review E. 85,066122, (2012).

[11] R. Albert and A. L. Barabási, "Reviews of Modern Physics" vol. 74, no. 47, (2002).

[12] C. Avin, B. Gurion, I. B. Sheva, "Distance graphs: from random geometric graphs to Bernoulli graphs and between", Proceedings of the fifth international workshop on Foundations of mobile computing, (2008).

[13] T. Zhou, G.Yan and B. H. Wang, Physical Review E. 71,046141, (2005).

[14] M. Bloznelis, Ann. Appl. Probab, vol. 23, no. 3, (2013).

[15] M. N. Kuperman and S. Risau-Gusman, Physical Review E. 86, 016104, (2012). 
International Journal of Future Generation Communication and Networking Vol. 9, No.7, (2016) 